\title{
Robotic-Assisted Radical Hysterectomy Results in Better Surgical Outcomes Compared With the Traditional Laparoscopic Radical Hysterectomy for the Treatment of Cervical Cancer
}

\author{
Ji-Chan Nie, MD, An-Qi Yan, MD, and Xi-Shi Liu, MD
}

\begin{abstract}
Objective: The aim of this study was to compare the surgical outcomes of robotic-assisted radical hysterectomy (RRH) with traditional laparoscopic radical hysterectomy (TLRH) for the treatment of early-stage cervical cancer in a large retrospective cohort of a total of 933 patients. Methods: We have enrolled 100 patients into the RRH and 833 patients into the TLRH group. The surgical outcomes include operating time, blood loss, transfusion rate, pelvic lymph node yield, hospitalization days, duration of bowel function recovery, catheter removal before and after 3 weeks, conversion to laparotomy, and intraoperative and postoperative complications. Follow-up results were also analyzed for all patients.

Results: Both groups have similar patient and tumor characteristics but patients with a larger lesion size were preferably enrolled in the TLRH treatment group. The treatment with RRH was generally superior to TLRH with respect to operating time, blood loss, length of hospitalization, duration of bowel function recovery, and postoperative complications. On follow-up of patients, there were no relapses reported in the RRH group compared with $4 \%$ of relapse cases and $2.9 \%$ of deaths because of metastasis in the TLRH group. No conversion of laparotomy occurred in the RRH group. No significant difference was found with respect to intraoperative complications and blood transfusion between both groups.

Conclusions: The results from this study suggest that RRH is superior to TLRH with regard to surgical outcome and may pose a safe and feasible alternative to TLRH. The operating time and lymph node yield is acceptable. Our study is one of the largest single-center studies of surgical outcomes comparing RRH with TLRH during cervical cancer treatment and will significantly contribute to the safety of alternative treatment options for patients. Furthermore, the difference detected between TLRH and RRH group is further strengthened by the great expertise of the surgeon performing laparoscopic surgeries.
\end{abstract}

Key Words: Robotic-assisted radical hysterectomy, Cervical cancer, Traditional laparoscopic radical hysterectomy

Received May 2, 2017, and in revised form June 30, 2017.

Accepted for publication July 11, 2017.

(Int J Gynecol Cancer 2017;27: 1990-1999)

Copyright (C) 2017 The Author(s). Published by Wolters Kluwer Health, Inc. on behalf of IGCS and ESGO. This is an open-access article distributed under the terms of the Creative Commons Attribution-Non Commercial-No Derivatives License 4.0 (CCBYNC-ND), where it is permissible to download and share the work provided it is properly cited. The work cannot be changed in any way or used commercially without permission from the journal. ISSN: $1048-891 \mathrm{X}$

DOI: $10.1097 /$ IGC.0000000000001101
Shanghai Obstetrics and Gynecology Hospital, Fudan University, Shanghai, China.

Address correspondence and reprint requests to Xi-Shi Liu, MD, Shanghai Obstetrics and Gynecology Hospital, Fudan University, Fangxie Rd, No. 419, Shanghai 200011, China. E-mail: liuxishi_lina@126.com. Ji-Chan Nie and An-Qi Yan are co-first author.

This research was supported by grant 81571416 (Jichan Nie) from the National Science Foundation of China.

The authors declare no conflicts of interest. 
$C^{2}$ ervical cancer is the second most common cancer in women according to the World Health Organization with an estimated number of 500,000 new cases and 250,000 deaths annually. ${ }^{1}$ The standard of surgical treatment of early-stage cervical cancer is the open radical hysterectomy with pelvic lymphadenectomy. ${ }^{2}$ In the early 90 s, minimally invasive surgery has become an acceptable alternative to open radical hysterectomy for the treatment of cervical cancer. ${ }^{3}$ The benefits of laparoscopy are less blood loss, decreased convalescence time, shorter hospital stay, decreased discomfort and superior wound healing compared with open radical hysterectomy. ${ }^{4-6}$ However, one of the drawbacks of the traditional laparoscopic radical hysterectomy (TLRH) is the long learning curve, 2-dimensional view, limited instruments movement, and poor ergonomics. ${ }^{7}$ In recent years, the robotic-assisted radical hysterectomy (RRH) became increasingly popular compared with TLRH because of the possibility of 3-dimensional vision and enhanced mobility of surgical instruments and shorter learning time. ${ }^{8,9}$ In 2005, the DaVinci Surgical system was approved for the RRH. ${ }^{10}$ Small comparative studies between open radical hysterectomy and TLRH or RRH have revealed that both techniques are safe in terms of surgical outcome and survival compared with the open radical hysterectomy, which is still the standard of treatment for cervical cancer. ${ }^{11-15}$ However, the number of studies comparing TLRH with RRH have been limited to small-sized studies with usually less than 50 patients in each study arm. ${ }^{16}$ Furthermore, most of the comparative studies on radical open hysterectomy have been conducted in the United States and in Europe. To the best of our knowledge, our study is the first comparative study of TLRH and RRH in cervical cancer patients in the mainland of China. Our cohort study is the largest single-center study comparing RRH cases $(n=100)$ with TLRH cases $(n=833)$ to date analyzing surgical outcome in early stage cervical cancer. Although many comparison studies exist for RRH and open radical hysterectomy, the aim of our study was to compare RRH with the TLRH procedure in patients with cervical cancer stages IA, IB, and IIA.

\section{MATERIALS AND METHODS}

\section{Patient and Tumor Characteristics}

A total of 933 patients underwent surgery for cervical cancer by a single surgeon during July 2009 and June 2016 at the Obstetrics and Gynecology Hospital of Fudan University, Shanghai, China. Of all cases, 833 patients had undergone a TLRH and 100 patients underwent an RRH. All patients were predominantly from the eastern region of China, and everybody consented to the type of surgery they received for the treatment of cervical cancer. Approval of this study was obtained from the institutional ethics review board of the Obstetrics and Gynecology Hospital of Fudan University. The surgeon and her team were certified by the Chinese University of Hong Kong Jockey Club Minimally Invasive Surgical Skills Centre for Robotic Surgery. The doctor has experience with TLRH surgeries since 2009 and with RRH since February 2015. Before 2009, only open radical hysterectomies were performed for the treatment of cervical cancer. The cervical cancer stage was determined according to the International Federation of Gynecology and Obstetrics. ${ }^{17}$ A Piver II/Type B surgery, for International Federation of Gynecology and Obstetrics (FIGO) stages IA1 and
IA2, or Piver III/type C surgery, for disease stages IB1, IB2, IIA1, and IIA2, was used for both surgical techniques according to the NCCN Clinical Practice Guidelines in Oncology National Clinical Practice Guidelines of Cancer. ${ }^{18}$

\section{Surgical Instruments and Procedure}

During the entire course of the laparoscopic surgery, the surgeon was positioned on a high stool. In our study, we used the EvisExera II CV-180 (Olympus, Tokyo, Japan) device for TLRH surgeries and the da Vinci Surgical System (Intuitive Surgical Inc, Sunnyvale, Calif) for the treatment with the RRH. Patients were first put under general endotracheal anesthesia and placed in a lithotomy-Trendelenburg position. The details of the surgical technique have been described in more details in previous studies. ${ }^{19-21}$ In brief, for TLRH, a 12-mm trocar was inserted into the umbilicus. Two 5-mm lateral trocars were placed symmetrically away from the umbilicus at a distance of $4 \mathrm{~cm}$ and below the horizontal-like of the umbilicus. The other 12-mm trocar was inserted bilaterally at the outer third of the iliac spine. For the robotic surgery, a total of 5 trocars were used. A 12-mm trocar was placed $4 \mathrm{~cm}$ away from the umbilicus for the camera. Two 8-mm trocars were placed $8 \mathrm{~cm}$ bilaterally to the umbilicus for the 2 robotic arms. In addition, a 5- and 12-mm trocar was placed in the left upper quadrant and the left outer third of the iliac spine umbilicus line for assistance, respectively. To expose the rectal- and vesical-vagina fold, an intrauterine manipulator was used for the uterus. For laparoscopy, a Harmonic Ace device was used (Ethicon, Cincinnati, Ohio), whereas for the RRH surgery a pair of monopolar scissors (Intuitive Surgical Inc) and fenestrated bipolar forceps (Intuitive Surgical Inc) were used. Pelvic lymphadenectomy was performed, and the ureter was dissected off the lateral peritoneum. For patients who opted for their ovaries to be removed, the infundibulopelvic ligament was cut, whereas for patients who opted to preserve their ovaries, the ovarian ligament was excised. The rectal vaginal peritoneal fold was exposed by manipulating the anterior part of the uterus. The uterine artery was cut, and the vessels were dissected over the ureter. The anterior and posterior vesico-uterine ligament on both sides were divided and incised. The cardinal and sacral ligament of the uterus were separated and dissected as previously described. ${ }^{22}$ The parametrial tissue was incised 1 to $2 \mathrm{~cm}$ from the margin or cut one fourth to one third of the vagina for types B and C radical hysterectomy, respectively. Biopsies were taken from the measured vaginal tissue. The vaginal cuff was then closed with a running locking suture. Monocryl sutures were used for sewing the peritoneum from underneath the bladder to the surface of the rectum.

\section{Parameters Studied and Statistical Analysis}

We analyzed the following parameters in our study: perioperative variables such as age, body mass index (BMI), cancer stages, and tumor characteristics, and intraoperative factors such as conversion to laparotomy, blood transfusion, blood loss, and operative time, and postoperative variables such as complications, recovery, length of hospitalization, and follow-up results. The presence of a Foley catheter as a consequence of surgery was assessed in all patients after 3 weeks postsurgery. Operative time was defined as the time from incision to closure 
of the wound. Blood loss was defined as the sum of suctioned fluids and weighted gauze minus the use of other fluids at the end of the surgery. For statistical analysis, we performed a Student $t$ test or Mann-Whitney $U$ test for continuous variables and $\chi^{2}$ test for binary data. A multivariate logistic regression model was used to evaluate associations of outcome and exposure. The odds ratio (OR) was calculated for binary data with a confidence interval (CI) of $95 \%$. The statistical $P$ value was calculated where appropriate with an $\alpha$ level of 0.05 . GraphPad software 6.0 was used to perform all statistical analysis.

\section{RESULTS}

A total of 933 patients were enrolled in our study of which 833 patients received a TLRH and 100 patients underwent a RRH for cervical cancer treatment. The median age was $47.1 \pm 9.49$ and $45.9 \pm 8.85$ years in the RRH and TLRH group, respectively (Fig. 1A; Table 1). There was no statistical difference in the mean BMI between both groups $\left(22.5 \pm 2.6 \mathrm{~kg} / \mathrm{m}^{2}\right.$ in the RRH group vs $22.6 \pm 2.3 \mathrm{~kg} / \mathrm{m}^{2}$ in the TLRH group) (Fig. 1B). The mean width of the cardinal ligament of the uterus was significantly higher in the RRH group ( $3 \pm$ $0.38 \mathrm{~cm})$ compared with the TLRH group $(2.8 \pm 0.63 \mathrm{~cm})$ lymphovascular space invasion was observed in $27 \%$ of RRH patients and $34.5 \%$ of TLRH patients. This difference was not statistically different $(P=0.69)$. The vaginal specimen length was similar in both groups (Table 1).

The cervical cancer stage was determined for each patient and categorized into stages according to the FIGO. In our study, we determined that $60 \%$ to $63.9 \%$ of patients had stage IB1, followed by $21 \%$ to $12 \%$ with stage IIA 1 in the RRH and TLRH group, respectively (Table 1). Only a few patients had stages IA2, IB2, and IIA2 disease (Fig. 2A). A type-II radical hysterectomy was only performed for stage IA2 cervical cancer, whereas type III was used for the other remaining stages of cancer (IB and IIA). Histological analysis of cancerous tissue was performed in all patients, and the type of tumor was identified in each group; in the RRH group: squamous cell carcinoma $(\mathrm{n}=72,72 \%)$, adenocarcinoma $(\mathrm{n}=27,27 \%)$, neuroendocrine carcinoma $(\mathrm{n}=1,1 \%)$ and in the TLRH group: squamous cell carcinoma $(\mathrm{n}=707,85 \%)$, adenocarcinoma $(\mathrm{n}=$ $111,13 \%)$, neuroendocrine carcinoma $(\mathrm{n}=12,1.4 \%)$, and adenosquamous cell carcinoma $(\mathrm{n}=3,0.4 \%)$ (Fig. 2B). The mean tumor lesion size of $2.5 \pm 1.02 \mathrm{~cm}$ in the RRH and $2.7 \pm$ $1.4 \mathrm{~cm}$ in the TLRH group was not statistically significant $(P=$ 0.12) (Fig. 2C). However, patients with tumor lesion sizes greater than $4 \mathrm{~cm}$ were significantly $(P=0.0008)$ more likely to receive laparoscopic radical hysterectomy compared with robotic-assisted surgery (Table 1). Tumor infiltration with bilateral uterine involvement or vaginal involvement was not statistically different between the RRH and TLRH groups $(\mathrm{n}=4,4 \%$ vs $\mathrm{n}=$ $31,3.7 \% ; P=0.88$ with bilateral uterine involvement and $\mathrm{n}=79$, $79 \%$ and $\mathrm{n}=744,89.3 \% ; P=0.025$ with bilateral vaginal involvement for RRH and TLRH groups, respectively). In the RRH group, $42 \%$ of cervical cancer patients had only superficial infiltration of the cervical stroma compared with $36 \%$ in the TLRH group. In $34 \%$ of patients in the RRH group, the tumor infiltrated the deep cervical muscle compared with $37 \%$ in the TLRH group. Metastasis was found in $27 \%$ of patients in the RRH group and in $34.5 \%$ of patients in the TLRH group and affected predominantly pelvic, iliac, and parametrial lymph nodes. This difference between both groups did not reach statistical significance.

To compare RRH with TLRH for the treatment of cervical cancer, we have analyzed the surgical outcome in all patients including operating time, blood loss, nodal count, length of hospitalization, blood transfusion, and intraoperative and postoperative complications (Table 2). The operative time in the RRH group was 20 minutes shorter compared with the TLRH group conditioned on blood loss and tumor stage in a multivariate regression analysis. This effect was highly significant with $95 \%$ confidence $(P<0.0005)$. The mean operative time was $171 \pm 38.84$ and $192.1 \pm 56.79$ minutes $(\mathrm{P}=0.0005)$ using robotic versus traditional laparoscopic surgery (Fig. 3A). The risk of blood loss increased significantly with the operating time during surgery $(P=0.00021)$ and was lower in the RRH group (Fig. 3B). The mean blood loss was significantly lower in the robotic group compared with the laparoscopic group (317.5 \pm
A

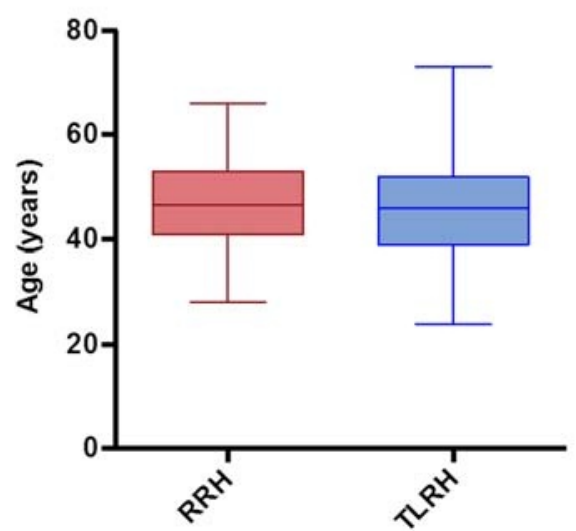

B

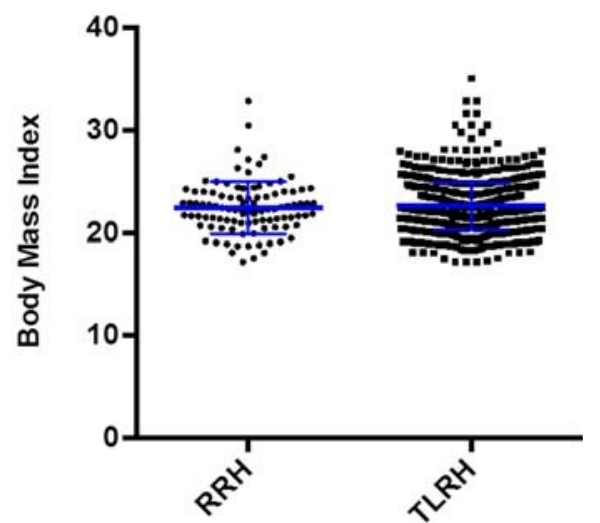

FIGURE 1. Patient characteristics undergoing either RRH (robotic) or TLRH treatment. A, Mean age \pm SD is shown. $B$, Mean $B M I \pm S D$ is shown. 
TABLE 1. Baseline patient and tumor characteristics of enrolled patients

\begin{tabular}{|c|c|c|c|}
\hline \multirow[b]{2}{*}{ Patients and Tumor Characteristics } & \multirow{2}{*}{$\begin{array}{c}\text { RRH } \\
n=100\end{array}$} & \multirow{2}{*}{$\begin{array}{c}\text { TLRH } \\
\mathrm{n}=\mathbf{8 3 3}\end{array}$} & \multirow[b]{2}{*}{$\boldsymbol{P}$} \\
\hline & & & \\
\hline Age, y & $47.1 \pm 9.49$ & $45.9 \pm 8.85$ & $0.19 *$ \\
\hline BMI, $\mathrm{kg} / \mathrm{m}^{2}$ & $22.5 \pm 2.6$ & $22.6 \pm 2.3$ & $0.55^{*}$ \\
\hline Cardinal ligament of uterus & $3.0 \pm 0.38$ & $2.8 \pm 0.63$ & $0.03 *$ \\
\hline Mean vaginal specimen length, $\mathrm{cm}$ & $2.8 \pm 0.48$ & $3.0 \pm 0.54$ & $0.05 *$ \\
\hline \multicolumn{4}{|l|}{ FIGO stage, $\mathrm{n}(\%)$} \\
\hline IA2 & $14(14)$ & $63(7.6)$ & \\
\hline IB1 & $60(60)$ & $532(63.9)$ & \\
\hline IB2 & $5(5)$ & 99 (11.9) & \\
\hline IIA1 & $21(21)$ & $100(12)$ & \\
\hline IIA2 & 0 & $38(4.6)$ & \\
\hline \multicolumn{4}{|l|}{ Histology, n (\%) } \\
\hline Adenocarcinoma & $27(27)$ & $111(13.3)$ & \\
\hline Neuroendocrine carcinoma & $1(1)$ & $12(1.4)$ & \\
\hline Adenosquamous cell carcinoma & 0 & $3(0.4)$ & \\
\hline Squamous cell carcinoma & $72(72)$ & $707(84.87)$ & \\
\hline \multicolumn{4}{|l|}{ Tumor lesion size, n (\%) } \\
\hline$<4 \mathrm{~cm}$ & $96(96)$ & $693(83.2)$ & \\
\hline$>4 \mathrm{~cm}$ & $5(5)$ & $137(16.4)$ & $0.0008^{*}$ \\
\hline Tumor lesion size, $\mathrm{cm}$ & $2.5 \pm 1.02$ & $2.7 \pm 1.4$ & $0.12 *$ \\
\hline Infiltration depth of cervical tumor, $\mathrm{n}(\%)$ & & & $0.0001 *$ \\
\hline No infiltration & 0 & $110(13.21)$ & \\
\hline Observed by the microscope & $14(14)$ & $28(3.36)$ & \\
\hline Superficial & $42(42)$ & $301(36.13)$ & \\
\hline Deep muscular & $34(34)$ & $310(37.21)$ & \\
\hline \multicolumn{4}{|l|}{ Tumor infiltration, n (\%) } \\
\hline Bilateral uterine involvement & $4(4)$ & $31(3.7)$ & $0.88^{*}$ \\
\hline Vaginal involvement & $79(79)$ & $744(89.3)$ & $0.025^{*}$ \\
\hline \multicolumn{4}{|l|}{ Metastasis, $\mathrm{n}(\%)$} \\
\hline Pelvic lymph node & $6(6)$ & $69(8.28)$ & $0.427^{*}$ \\
\hline Parametrial lymph node & $4(4)$ & $78(9.36)$ & $0.07 *$ \\
\hline Iliac & $5(5)$ & $29(3.48)$ & $0.44^{*}$ \\
\hline Obturator lymph node & $5(5)$ & $73(8.7)$ & $0.26^{*}$ \\
\hline Lymphovascular space invasion, $\mathrm{n}(\%)$ & $27(27)$ & $288(34.57)$ & $0.669^{*}$ \\
\hline
\end{tabular}

144.2 vs $322.5 \pm 178 \mathrm{~mL} ; P=0.015$ ) (Fig. 3C). In addition to that, the length of hospitalization was 1 day shorter in the RRH group compared with the TLRH group. This difference reached statistical significance with an $\alpha$ level of $0.05 \%(10.4 \pm 2.74$ days RRH vs $11.5 \pm 3.94$ days TLRH; $P=0.009$ ) (Fig. 3D). An overall mean of 22.4 pelvic lymph nodes were counted in each patient, and the ovaries were excised in most of the patients (61\% in the RRH group and $56.3 \%$ in the TLRH group). Blood transfusion was significantly more often administered to the
RRH group $(P=0.048)$ compared with the TLRH group. Eleven percent of the robotically treated and $5.9 \%$ of the traditional laparoscopically treated patients received blood transfusion. The catheter was removed in $97 \%$ of RRH patients within 3 weeks after surgery and in significantly fewer patients $(88.8 \%)$ of the TLRH group $(P=0.011)$. In addition to that, the duration of bowel function recovery was faster in the robotic group compared with the traditional laparoscopic group $(1.11 \pm 0.31 \mathrm{vs}$ $2.06 \pm 0.54$ days; $P=0.0001$, respectively) (Fig. 3E). 
A

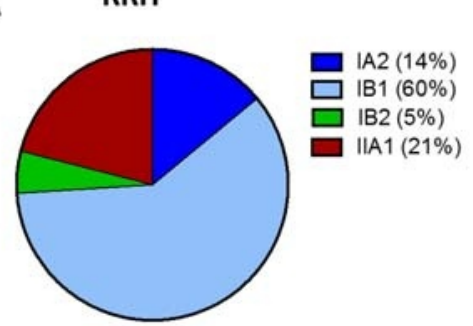

C

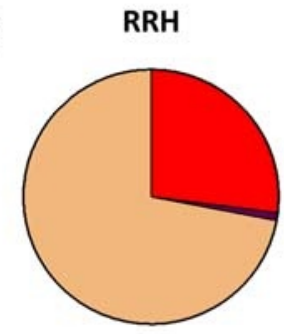

E

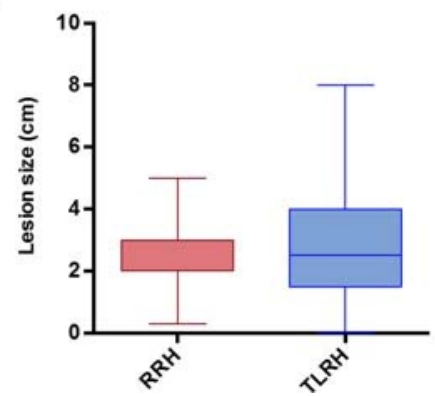

B TLRH

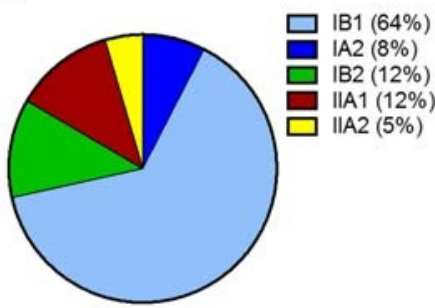

D

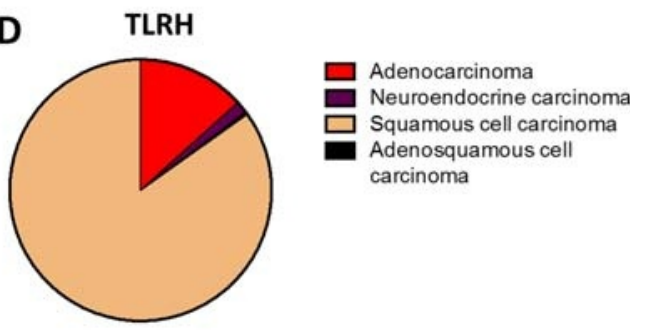

FIGURE 2. Tumor characteristics of all study participants. A, Proportions (\%) of patients in the RRH (left) and TLRH (right) with different cervical cancer stages within each group ( $n=100$ for RRH and $n=833$ for TLRH). B, Proportions (\%) of type of cancer within the RRH (left) and TLRH group (right). C, Size of tumor lesion (centimeter) identified in each patient. Error bars depict distribution of patients with minimum and maximum values.

Postoperative adjuvant radiotherapy was given to $40 \%$ and $55.3 \%$ of patients in the RRH and TLRH group, respectively. This difference was not significant $(P=0.09)$ comparing both groups. Conversion to laparotomy because of intraoperative complications occurred only in 3 patients of the TLRH group. Thus, after analyzing several surgical outcomes, robotic-assisted cervical hysterectomy is superior to the traditional laparoscopic surgery for the treatment of early-stage cervical cancer.

Next, we analyzed intraoperative and postoperative complications within both groups of our cohort study (Table 3). We found that the odds of having intraoperative and postoperative complications in the robotic group were 0.28 and 0.35 times of those with laparoscopic surgery, respectively (intraoperative complications: OR 0.28 [CI, 0.02-4.75] and postoperative complications: OR 0.35 [CI, 0.12-0.99]). Of note, no intraoperative complications occurred in the RRH group compared with 14 cases in the TLRH group (Table 3). Of these patients, 7 patients had a major vessel injury, and 7 patients had bladder injury during the laparoscopic surgery. Postoperative complications occurred in $4 \%$ and $13.2 \%$ of robotic and laparoscopic patients, respectively (Table 3 ). This difference reached statistical significance $(P=0.039)$. Within the RRH group, there was 1 patient who developed ureterovaginal fistula and 3 patients with hydronephrosis. Within the TLRH group, we reported 30 patients with ureterovaginal fistula, 44 patients with hydronephrosis, 3 patients with lymphedema, 12 patients with remnant drain catheter, and 4 patients with severe leg edema and pain. Follow-up of patients within our cohort revealed no relapses within the robotic group and 32 cases with relapse in the laparoscopic group (Table 2). This difference was statistically different (OR, 0.07 [CI, 0.004-0.99]; $P=0.009$ ). In brief, within the TLRH group, $4.2 \%$ of patients experienced a relapse, $2.9 \%$ died because of metastasis, and $2.1 \%$ were lost to followup. Thus, we have shown in this study that for the treatment of cervical cancer, the robotic-assisted type of surgery was beneficial compared with the traditional laparoscopic surgery with fewer intraoperative and postoperative complications, shorter operating time, decreased blood loss, reduced length of hospitalization, and better long-term outcomes during follow-up visits.

\section{DISCUSSION}

Few studies have suggested that minimally invasive surgery and RRH are feasible and safe options for the treatment of 
TABLE 2. Surgical outcomes and operative findings

\begin{tabular}{|c|c|c|c|c|c|}
\hline & RRH & TLRH & & & \\
\hline & $\mathbf{n}=100$ & $\mathbf{n}=\mathbf{8 3 3}$ & $\boldsymbol{P}$ & OR & CI \\
\hline Operative time, $\min$ & $171.64 \pm 38.84$ & $192.10 \pm 56.79$ & $0.0005^{*}+$ & & \\
\hline Blood loss, $\mathrm{mL}$ & $317.5 \pm 144.20$ & $322.51 \pm 178.00$ & $0.015^{*}+$ & & \\
\hline Hospitalization, $\mathrm{d}$ & $10.41 \pm 2.74$ & $11.5 \pm 3.94$ & $0.09 * t$ & & \\
\hline \multicolumn{6}{|l|}{ Ovaries, n (\%) } \\
\hline Retained & $39(39)$ & $360(43.2)$ & $0.39 *$ & 0.83 & $0.54-1.27$ \\
\hline Excised & $61(61)$ & $469(56.3)$ & & & \\
\hline Blood transfusion, $\mathrm{n}(\%)$ & $11(11)$ & $49(5.9)$ & $0.048 \dagger t$ & 1.98 & $1.00-3.94$ \\
\hline Catheter removal within 3 wks postsurgery, n (\%) & $97(97)$ & $740(88.84)$ & $0.0111+t$ & 0.25 & $0.08-0.79$ \\
\hline Lymph nodes counted, $\mathrm{n}$ & $22.39 \pm 3.91$ & $22.51 \pm 5.19$ & $0.81 *$ & & \\
\hline Duration of bowel function recovery, $\mathrm{d}$ & $1.11 \pm 0.31$ & $2.06 \pm 0.54$ & $0.0001 \dagger t$ & & \\
\hline Postoperative adjuvant therapy, $\mathrm{n}(\%)$ & $40(40)$ & $461(55.34)$ & $0.0955 \dagger$ & 0.43 & $0.15-1.20$ \\
\hline Conversion to laparotomy, n (\%) & 0 & $3(0.33)$ & $0.54 \dagger$ & 1.18 & $0.06-23$ \\
\hline \multicolumn{6}{|l|}{ Follow-up visit result } \\
\hline No relapse & $100(100)$ & $756(90.8)$ & $0.009+t$ & 0.07 & $0.004-0.99$ \\
\hline Relapse & 0 & $32(4.2)$ & $0.03 \dagger t$ & 0.11 & $0.007-0.98$ \\
\hline Death & 0 & $24(2.9)$ & $0.07 \dagger$ & 0.15 & $0.009-2.47$ \\
\hline
\end{tabular}

early stage cervical cancer. ${ }^{16,20}$ In contrast to this, other studies have demonstrated that no significant benefits exist comparing the RRH and TLRH procedure. ${ }^{23,24}$ Based on these contradictory findings larger studies are required to compare $\mathrm{RRH}$ with TLRH treatment to evaluate the benefits of this expensive surgical procedure. ${ }^{25,26}$ Our retrospective cohort study is one of the largest single-center studies of surgical and oncologic outcomes comparing RRH and TLRH and is the first study comparing both procedures in Chinese patients. A total of 100 and 833 patients were enrolled in the RRH and TLRH group for the treatment of early stage cervical cancer, respectively. A recent study by Wallin et $\mathrm{al}^{26}$ analyzed 149 cases of RRH but these were compared with open radical hysterectomy and not to TLRH as conducted in our study. Of all 11 published studies comparing RRH with TLRH for the treatment of cervical cancer, the largest study included only 73 patients that underwent RRH surgery compared with 46 patients that opted for a TLRH procedure. ${ }^{27}$ Although many of those studies include only stages IA and IB cervical cancer patients, we have also enrolled patients with stage IIA in our study. Similar to previously published studies, most of the patients had a squamous cell carcinoma but other tumor types were also identified in both groups including adenocarcinoma, neuroendocrine carcinoma, and adenosquamous cell carcinoma. The TLRH procedure was preferred over RRH for patients with a tumor lesion size greater than $4 \mathrm{~cm}$ and for tumors that infiltrated into the deep muscle of the cervix. This preference over RRH is probably because of an increased experience and familiarity of the surgeon with the TLRH procedure compared with RRH surgery. For both techniques, the learning curve is steep and involves many hours of practice to become proficient in instrument assembly and use of the robot arms. ${ }^{19,28}$ However, the surgeon performing the laparoscopic surgeries in this study has an exceptional proficiency in this technique because of the exceptionally large number of patients treated per year underlining the significance of our findings that RRH is superior to TLRH.

Our study was performed by a single surgeon at a singlecenter study site, which facilitates comparisons between the 2 treatment groups. A recent multi-institutional study from the United States has shown that the operating time and blood loss in both groups was significantly variable between both groups and compared with the open radical hysterectomy group depending on the surgeon's proficiency in both surgical techniques. ${ }^{29}$ Regarding patient characteristics, there was no difference in mean age and BMI between both treatment groups in accordance with other similar studies. The mean age was $47 \pm 9.49$ and $46 \pm 8.85$ years in the RRH and TLRH group, respectively. Our main findings reveal that the RRH group was generally better in regard to surgical outcome compared with the RRH group. In support of this, several other studies have found that blood loss and the length of hospitalization were significantly reduced when undergoing an RRH surgery. ${ }^{20,26,30}$ Contrary to this result, another study reported no benefit of RRH over TLRH treatment for cervical cancer patients. ${ }^{13}$ In comparison with previously published reports, we have looked at many 
A

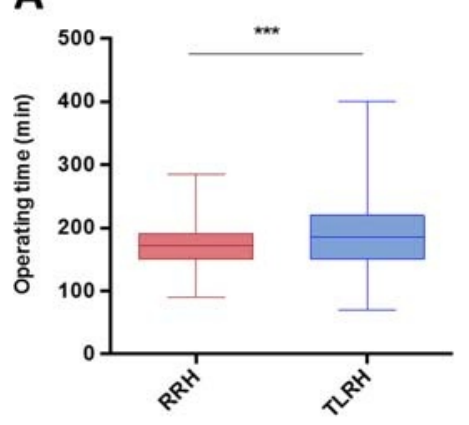

C

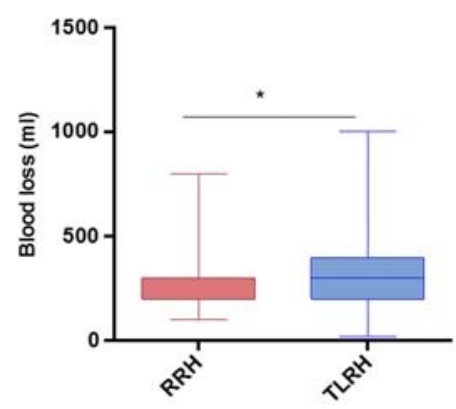

E

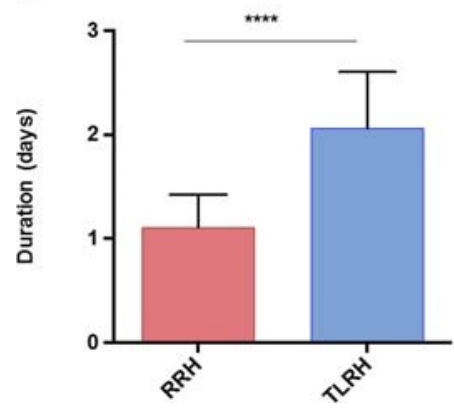

B

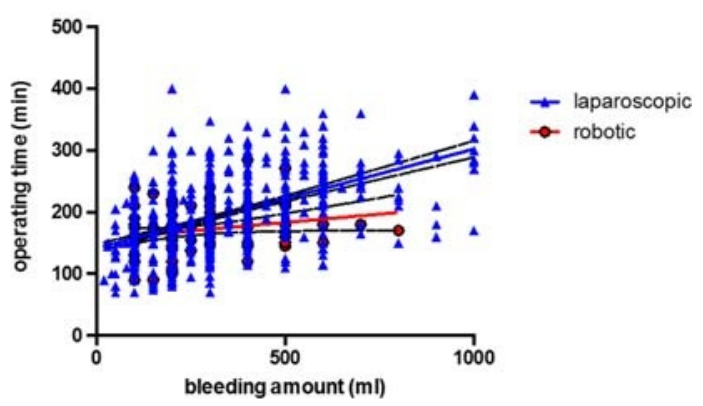

D

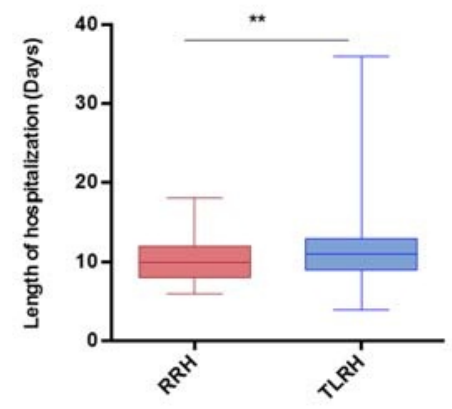

FIGURE 3. Surgical outcome of RRH and TLRH treatment of cervical cancer. A, Operating time (minutes). B, Linear regression of blood loss compared with operating time with a $95 \% \mathrm{Cl}$ in each group. C, Blood loss (milliliter). D, Length of hospitalization (days). E, Duration of bowel function recovery (days). For box whisker plots, the distribution with minimum and maximum values is shown as error bars. For bar graphs, the mean \pm SD is shown. A Student $t$ test was performed with ${ }^{*} P<0.05,{ }^{* *} P<0.01$, and ${ }^{* * *} P<0.001$.

surgical outcomes in our study including, operating time, blood loss, removal of catheter after 3 weeks postsurgery, pelvic lymph node count, duration of bowel recovery, conversion to laparotomy, length of hospital stay, intraoperative and postoperative complications and recurrence of tumors.

We have shown that the mean operating time in the RRH group was significantly reduced by approximately 20 minutes compared with the TLRH group (171.6 \pm 38.84 vs $192 \pm 56.79$ minutes, respectively). The operating time in the RRH group was generally quite fast compared with the operating time documented in similar published studies. The shortest operating time was reported by 2 previously published studies with a mean time of 144 and 185 minutes. ${ }^{31,32}$ In contrast to this, there were also several other studies that had a much longer operating time in the RRH group compared with our study. ${ }^{11,22}$ Thus, the duration of the operating time is quite variable as the definition of "operating time" differs between studies. ${ }^{7}$ Therefore, the lack of standardization of the term operating time makes it complicated to draw comprehensive conclusions from this outcome alone. A significant factor that contributes to an enhanced operating time is each surgeon's previous experience with TLRH and RRH surgery. Both TLRH and RRH require an increased operating time compared with the traditional open radical hysterectomy because the surgeons have to familiarize themselves first with the instrument assembly, docking, and console time. One study suggests that a minimum of $20 \mathrm{RRH}$ surgeries are required to significantly reduce the operating time over time. ${ }^{28}$ Contrary to this authors from another 2 studies 
TABLE 3. Intraoperative and postoperative complications in each group

\begin{tabular}{|c|c|c|c|c|}
\hline & RRH & TLRH & & \\
\hline & $\overline{n=100}$ & $\mathrm{n}=\mathbf{8 3 3}$ & OR & CI \\
\hline Intraoperative complications $\mathrm{n}(\%)$ & 0 & $14(1.6)$ & 0.28 & $0.02-4.75$ \\
\hline Major vessel injury & 0 & $7(0.84)$ & 0.548 & $0.03-9.678$ \\
\hline Bladder injury & 0 & $7(0.84)$ & 0.548 & $0.032-9.678$ \\
\hline Postoperative complications, n (\%) & $4(4)$ & $110(13.2)$ & 0.3553 & $0.12-0.9914$ * \\
\hline Ureterovaginal fistula & $1(1)$ & $30(3.6)$ & 0.1811 & $0.0246-1.33$ \\
\hline Hydronephrosis & $3(3)$ & $44(5.2)$ & 0.55 & $1.689-1.821$ \\
\hline Lymphedema & 0 & $3(0.33)$ & 1.18 & $0.065-23.04$ \\
\hline Remnant drain catheter & 0 & $12(1.44)$ & 0.327 & $0.0192-5.568$ \\
\hline Server leg edema/pain & 0 & $4(0.48)$ & 0.917 & $0.048-17.17$ \\
\hline
\end{tabular}

Data are presented as percentage (\%) of the total number (n). The OR and the $95 \%$ CI were calculated.

$* P<0.05$ vs. the RRH.

argue that no previous experience is needed to reduce the operating time. ${ }^{29,33}$ Thus, it remains unclear if previous experience with RRH will decrease operating time.

We have also shown that blood loss is significantly reduced in the RRH group compared with the TLRH group. This is in accordance with 2 other studies that have shown that blood loss is significantly decreased in the RRH group compared with the TLRH group. ${ }^{22,34}$ In addition to that, blood loss was highest when using traditional radical hysterectomy surgery with a mean blood loss of 450 to $1000 \mathrm{~mL} .^{11,30,32,33}$ The reason for a decrease in blood loss during RRH surgery compared with TLRH is that the sacral afferent fibers and deep uterine veins can be more easily differentiated because of the robotic arm. In addition, the movement of the robotic arm results in stable traction and counter traction and excellent 3-dimensional view within a narrow space. ${ }^{19,22}$ A recent meta-analysis revealed that the blood loss in the RRH group can be as low as 78 or 82 mL. ${ }^{16,35,36}$ In our study, the mean blood loss was much higher compared with those studies but was still significantly lower in the RRH group versus the TLRH group. However, it remains unclear why the RRH group required more blood transfusions during surgery compared with the TLRH group. Although more patients in the RRH group required blood transfusion compared with the TLRH group, all the other 89 of 100 patients in the RRH group were doing much better compared with the TLRH group in terms of blood loss and lost significantly less volume of blood compared with the TLRH group. The 11 patients who received the blood transfusion had a more advanced stage of cervical cancer, higher operating time compared with the mean operating time, and were older compared with the other patients in the RRH group.

Intraoperative and postoperative complications were decreased in the RRH group compared with the TLRH group, and no conversions to laparotomy were reported in our RRH patient group. In addition, RRH and TLRH procedures result in fewer intraoperative and postoperative complications compared with the traditional radical hysterectomy. ${ }^{7,16,20}$ Similar to blood loss, the number of complications within each group is highly variable and dependent on previous experience of the surgeon with laparoscopy.

The length of hospital stay was significantly 1 day shorter in the RRH group compared with the TLRH group with a mean hospitalization time of 10 to 11 days in both groups. This is in contrast to a few other recent studies where the length of hospitalization was reported to be between 1.7 and 3 days. ${ }^{14,34}$ Interestingly, we noticed that studies from the United States tend to have much shorter duration of hospitalization compared with all laparoscopic studies conducted in Asia including 3 studies from Korea and 1 from Taiwan. ${ }^{11,12,37}$ Maybe this is because of societal differences, because cancer patients in Asia tend to stay longer in the hospital because of national health insurance subsidies as suggested by Chen et al. ${ }^{11}$

The dissection of pelvic lymph nodes during radical hysterectomy is critical for the outcome of the patient. ${ }^{38-40}$ Although only a mean of 9 lymph nodes was dissected using the traditional radical hysterectomy, an average of 20 lymph nodes was excised during TLRH or RRH surgery. ${ }^{30,41}$ In our study, we showed that the mean count of lymph nodes in both groups was 22.3 lymph nodes. No difference was observed between the RRH and TLRH group. The increase in lymph node counts that are dissected during RRH or TLRH procedures compared with the traditional hysterectomy is due to an increased precision with the laparoscope or robotic arm and is beneficial for the patient. ${ }^{11}$

Lastly, we also assessed the frequency of relapse or deaths in RRH and TLRH treated patients. Our results reveal that the follow-up was significantly better in the RRH group compared with the TLRH group with no reported relapses in the robotic arm. However, this result has to be interpreted cautiously because the TLRH group contained more severe cases of cervical cancer compared with the RRH group in terms of tumor infiltration and size. One recent study has demonstrated that the recurrence rate in the RRH group was higher compared with the TLRH group. ${ }^{26}$ However, this result may also be because of the experience of the surgeon with the RRH or TLRH procedure. Thus, structured training and monitoring outcomes are 
pivotal when introducing new treatment modalities. One limitation of our study was the lack of randomization and long-term follow-up studies.

In conclusion, we have shown in our study that RRH is superior to TLRH for the treatment of early-stage cervical cancer but the outcomes were generally quite similar in both groups. Although the cost of RRH is initially high for each hospital, the price per patient will drop with increased surgeon experience, shorter length of hospitalization, and increased number of RRH surgeries. ${ }^{25,26}$ This will eventually outweigh the benefits over costs. Our findings contribute to the field of robotic versus laparoscopic radical hysterectomy in terms of safety and feasibility, and we have demonstrated that the use of RRH is a safe and beneficial alternative to TLRH.

\section{REFERENCES}

1. Parkin DM, Bray F, Ferlay J, et al. Estimating the world cancer burden: Globocan 2000. Int J Cancer. 2001;94:153-156.

2. Averette HE, Nguyen HN, Donato DM, et al. Radical hysterectomy for invasive cervical cancer. A 25 -year prospective experience with the Miami technique. Cancer. 1993;71: 1422-1437.

3. Nezhat CR, Burrell MO, Nezhat FR, et al. Laparoscopic radical hysterectomy with paraaortic and pelvic node dissection. Am J Obstet Gynecol. 1992;166:864-865.

4. Steed H, Rosen B, Murphy J, et al. A comparison of laparascopic-assisted radical vaginal hysterectomy and radical abdominal hysterectomy in the treatment of cervical cancer. Gynecol Oncol. 2004;93:588-593.

5. Jackson KS, Das N, Naik R, et al. Laparoscopically assisted radical vaginal hysterectomy vs. radical abdominal hysterectomy for cervical cancer: a match controlled study. Gynecol Oncol. 2004;95:655-661.

6. Sharma R, Bailey J, Anderson R, et al. Laparoscopically assisted radical vaginal hysterectomy (Coelio-Schauta): a comparison with open Wertheim/Meigs hysterectomy. Int J Gynecol Cancer. 2006;16:1927-1932.

7. Renato S, Mohamed M, Serena S, et al. Robot-assisted radical hysterectomy for cervical cancer: review of surgical and oncological outcomes. ISRN Obstet Gynecol. 2011;2011: 872434.

8. Park DA, Yun JE, Kim SW, et al. Science direct. Eur J Surg Oncol. 2017:1-9.

9. Magrina JF. Robotic surgery in gynecology. Eur J Gynaecol Oncol. 2007;28:77-82.

10. Holloway RW, Patel SD, Ahmad S. Robotic surgery in gynecology. Scand J Surg. 2009;98:96-109.

11. Chen $\mathrm{CH}$, Chiu $\mathrm{LH}$, Chang $\mathrm{CW}$, et al. Comparing robotic surgery with conventional laparoscopy and laparotomy for cervical cancer management. Int J Gynecol Cancer. 2014;24:1105-1111.

12. Yim GW, Kim SW, Nam EJ, et al. Surgical outcomes of robotic radical hysterectomy using three robotic arms versus conventional multiport laparoscopy in patients with cervical cancer. Yonsei Med J. 2014;55:1222-1230.

13. Tinelli R, Malzoni M, Cosentino F, et al. Robotics versus laparoscopic radical hysterectomy with lymphadenectomy in patients with early cervical cancer: a multicenter study. Ann Surg Oncol. 2011;18:2622-2628.

14. Nezhat F. Minimally invasive surgery in gynecologic oncology: laparoscopy versus robotics. Gynecol Oncol. 2008;111: S29-S32.
15. Sert B, Abeler V. Robotic radical hysterectomy in early-stage cervical carcinoma patients, comparing results with total laparoscopic radical hysterectomy cases. The future is now? Int J Med Robot. 2007;3:224-228.

16. Shazly SA, Murad MH, Dowdy SC, et al. Robotic radical hysterectomy in early stage cervical cancer: a systematic review and meta-analysis. Gynecol Oncol. 2015;138:457-471.

17. Pecorelli S. Revised FIGO staging for carcinoma of the vulva, cervix, and endometrium. Int J Gynaecol Obstet. 2009; 105:103-104.

18. Koh WJ, Greer BE, Abu-Rustum NR, et al. Cervical cancer, version 2.2015. J Natl Compr Canc Netw. 2015;13:395-404.

19. Chong GO, Park NY, Hong DG, et al. Learning curve of laparoscopic radical hysterectomy with pelvic and/or para-aortic lymphadenectomy in the early and locally advanced cervical cancer: comparison of the first 50 and second 50 cases. Int J Gynecol Cancer. 2009;19:1459-1464.

20. Park NY, Chong GO, Hong DG, et al. Oncologic results and surgical morbidity of laparoscopic nerve-sparing radical hysterectomy in the treatment of FIGO stage IB cervical cancer: long-term follow-up. Int J Gynecol Cancer. 2011;21:355-362.

21. Lee YS, Chong GO, Lee YH, et al. Robot-assisted total preservation of the pelvic autonomic nerve with extended systematic lymphadenectomy as part of nerve-sparing radical hysterectomy for cervical cancer. Int J Gynecol Cancer. 2013;23:1133-1138.

22. Chong GO, Lee YH, Hong DG, et al. Robot versus laparoscopic nerve-sparing radical hysterectomy for cervical cancer: a comparison of the intraoperative and perioperative results of a single surgeon's initial experience. Int J Gynecol Cancer. 2013:23:1146-1150.

23. Antosh DD, Grotzke SA, McDonald MA, et al. Short-term outcomes of robotic versus conventional laparoscopic sacral colpopexy. Female Pelvic Med Reconstr Surg. 2012;18: 158-161.

24. Paraiso MF, Ridgeway B, Park AJ, et al. A randomized trial comparing conventional and robotically assisted total laparoscopic hysterectomy. Am J Obstet Gynecol. 2013:208:368.e1-7.

25. Reynisson P, Persson J. Hospital costs for robot-assisted laparoscopic radical hysterectomy and pelvic lymphadenectomy. Gynecol Oncol. 2013;130:95-99.

26. Wallin E, Flöter Rådestad A, Falconer H. Introduction of robot-assisted radical hysterectomy for early stage cervical cancer: impact on complications, costs and oncologic outcome. Acta Obstet Gynecol Scand. 2017;36:536-542.

27. Gortchev G, Tomov S, Tantchev L, et al. Robot-assisted radical hysterectomy-perioperative and survival outcomes in patients with cervical cancer compared to laparoscopic and open radical surgery. Gynecol Surg. 2011;9:81-88.

28. Ko EM, Muto MG, Berkowitz RS, et al. Robotic versus open radical hysterectomy: a comparative study at a single institution. Gynecol Oncol. 2008;111:425-430.

29. Lowe MP, Chamberlain DH, Kamelle SA, et al. A multi-institutional experience with robotic-assisted radical hysterectomy for early stage cervical cancer. Gynecol Oncol. 2009;113:191-194.

30. Soliman PT, Frumovitz M, Sun CC, et al. Radical hysterectomy: a comparison of surgical approaches after adoption of robotic surgery in gynecologic oncology. Gynecol Oncol. 2011;123:333-336.

31. Corrado G, Fanfani F, Ghezzi F, et al. Mini-laparoscopic versus robotic radical hysterectomy plus systematic pelvic 
lymphadenectomy in early cervical cancer patients.

A multi-institutional study. Eur J Surg Oncol. 2015;41:136-141.

32. Estape R, Lambrou N, Diaz R, et al. A case matched analysis of robotic radical hysterectomy with lymphadenectomy compared with laparoscopy and laparotomy. Gynecol Oncol. 2009;113:357-361.

33. Boggess JF, Gehrig PA, Cantrell L, et al. A case-control study of robot-assisted type III radical hysterectomy with pelvic lymph node dissection compared with open radical hysterectomy. Am J Obstet Gynecol. 2008;199:357.e1-7.

34. Magrina JF, Kho RM, Weaver AL, et al. Robotic radical hysterectomy: comparison with laparoscopy and laparotomy. Gynecol Oncol. 2008;109:86-91.

35. Maggioni A, Minig L, Zanagnolo V, et al. Robotic approach for cervical cancer: comparison with laparotomy: a case control study. Gynecol Oncol. 2009;115:60-64.

36. Sert MB, Abeler V. Robot-assisted laparoscopic radical hysterectomy: comparison with total laparoscopic hysterectomy and abdominal radical hysterectomy; one surgeon's experience at the Norwegian Radium Hospital. Gynecol Oncol. 2011;121:600-604.
37. Kim TH, Choi $\mathrm{CH}$, Choi JK, et al. Robotic versus laparoscopic radical hysterectomy in cervical cancer patients. Int $J$ Gynecol Cancer. 2014;24:1466-1473.

38. Gold MA, Tian C, Whitney CW, et al. Surgical versus radiographic determination of para-aortic lymph node metastases before chemoradiation for locally advanced cervical carcinoma: a Gynecologic Oncology Group Study. Cancer. 2008;112:1954-1963.

39. Keys HM, Bundy BN, Stehman FB, et al. Cisplatin, radiation, and adjuvant hysterectomy compared with radiation and adjuvant hysterectomy for bulky stage IB cervical carcinoma. N Engl J Med. 1999;340:1154-1161.

40. Morris M, Eifel PJ, Lu J, et al. Pelvic radiation with concurrent chemotherapy compared with pelvic and para-aortic radiation for high-risk cervical cancer. $N$ Engl J Med. 1999;340: 1137-1143.

41. Desille-Gbaguidi H, Hebert T, Paternotte-Villemagne J, et al. Overall care cost comparison between robotic and laparoscopic surgery for endometrial and cervical cancer. Eur J Obstet Gynecol Reprod Biol. 2013;171:348-352. 